\title{
Gestão das solicitações de medicamentos não padronizados em um hospital universitário
}

\author{
Ana Cristina Batista Lino*, Fernanda Gomes Nascimento, \\ Danielle Cristina Rodrigues Vieira, Gisele Zanetti Senhorin
}

Setor de Farmácia do Hospital Universitário Dr. Miguel Riet Correa Jr, Rio Grande, RS, Brasil

\section{Histórico do Artigo \\ Recebido em: \\ 10/04/2019 \\ Aceito em: \\ $16 / 03 / 2020$}

Palavras-chave:

Assistência farmacêutica;

Serviço de Farmácia

Hospitalar; qualidade

da Assistência à

Saúde.

Keywords:

Pharmacy assistance; Hospital Pharmacy Service; Quality of Health Care

\begin{abstract}
RESUMO
A padronização dos medicamentos em hospitais é um processo de escolha baseado em critérios epidemiológicos, técnicos e econômicos, estabelecidos por uma Comissão de Farmácia e Terapêutica (CFT). Entretanto, nem sempre o elenco dos medicamentos padronizados é suficiente para atender demandas excepcionais, por isso, é importante dispor de um método que assegure o uso de medicamentos não padronizados. O presente trabalho trata de um estudo descritivo, retrospectivo caracterizado como exploratório e realizado através da análise de 153 formulários de solicitações de medicamentos não padronizados enviados ao setor de farmácia do Hospital Universitário no período de agosto a 31 de dezembro de 2018. Os dados coletados foram analisados no programa Excel. Foram analisados 153 formulários de solicitações de medicamentos não padronizados, com uma média aproximada de 30 solicitações/mês e um total de 72 medicamentos diferentes. O medicamento alprazolam (1mg) comprimido com $11(7,19 \%)$ solicitações é um candidato à inclusão na lista de padronização. A maioria dos pedidos $110(71,9 \%)$, foram atendidos. Outras $13(8,50 \%)$ solicitações sofreram intervenções pelos farmacêuticos. A Clínica Médica que atende um público heterogêneo e com patologias diversas foi a clínica que mais solicitou medicamentos não padronizados $(37,25 \%)$. Com a elaboração de uma lista de medicamentos não padronizados é possível revisar a padronização e sugerir a inclusão de novos medicamentos. Também se concluiu que o farmacêutico possui ação relevante na análise destas solicitações porque garante o uso racional dos medicamentos e o gerenciamento de novas aquisições, dentro do orçamento do hospital.
\end{abstract}

Management of requests for non-standard medication in a university hospital

\section{ABSTRACT}

The standardization of medicines in hospitals is a choice process based on epidemiological, technical and economic criteria, established by a Pharmacy and Therapeutic Committee (CFT). However, the list of standardized medicines is not always sufficient to meet exceptional demands, so it is important to have a method that ensures the use of non-standard medicines. The present study is a descriptive, retrospective study characterized as exploratory and carried out through the analysis of 153 non-standardized medication order forms sent to the pharmacy sector of the University Hospital during August to December 31, 2018. The collected data were analyzed in the Excel program and expressed in graphs and tables. 153 forms of non-standardized medication requests were analyzed, with an average of approximately 30 requests / month and a total of 72 different medications. The drug alprazolam $1 \mathrm{mg}$ tablet with $11(7.19 \%)$ requests is a candidate for inclusion in the standardization list. The majority of requests 110 (71.9\%), were answered. Another $13(8.50 \%)$ requests suffered interventions by pharmacists. The Medical Clinic that serves a heterogeneous public with different pathologies was the clinic that most requested non-standard drugs. With the elaboration of a list of non-standardized drugs it is possible to review the standardization and suggest the inclusion of new drugs. It was also concluded that the pharmacist has relevant action in the analysis of these requests because it guarantees the rational use of medicines and the management of new acquisitions, within the hospital's budget.

\section{Introdução}

Diante de um número cada vez maior de medicamentos e da constante inovação tecnológica presente no mercado, o cuidado com a seleção de medicamentos

\footnotetext{
*Autor correspondente: lino.ana@gmail.com (Lino A.C.B.)
} 
padronizados dentro de uma instituição hospitalar torna-se essencial. A padronização de medicamentos tem como objetivo o uso racional dos medicamentos, com seleção de produtos de valor terapêutico comprovados, a diminuição do número de medicamentos em estoque, o aumento do controle e agilidade da dispensação destes e proporcionar maior eficiência administrativa para a alocação de recursos para a aquisição dos insumos farmacêuticos $(1,2)$.

A Organização Mundial de Saúde (OMS) sugere que as listas nacionais de medicamentos essenciais sejam bases para as escolhas dos tratamentos terapêuticos, protocolos e diretrizes das instituições ligadas ao Sistema Único de Saúde (SUS) (3). Os medicamentos essenciais são definidos pela OMS como aqueles que satisfazem as necessidades de atenção à saúde da maioria da população (4).

A padronização dos medicamentos dentro da instituição hospitalar é um processo de escolha baseado em critérios epidemiológicos, técnicos e econômicos, estabelecidos por uma Comissão de Farmácia e Terapêutica (CFT) (3). Visa uniformizar a terapêutica dos pacientes e disciplinar a prescrição médica sempre que possível (1).

A CFT é uma instância colegiada, de caráter consultivo e deliberativo, atuando como ponto fundamental na busca pela eficiência na utilização dos recursos em saúde (5). Entretanto, nem sempre o elenco dos medicamentos padronizados é suficiente para atender demandas excepcionais, por isso, é importante dispor de um método que assegure o uso de medicamentos não padronizados (3). Uma ferramenta utilizada é o formulário de solicitação de medicamentos não padronizados preenchido por médicos e residentes que realizam atendimentos, principalmente em instituição de alta complexidade.

Segundo a Resolução CIT/MS n ${ }^{\circ} 29$, o formulário para justificativa de prescrição de medicamentos não padronizados no SUS, a ser preenchido pelo prescritor, deverá indicar e justificar, do ponto de vista clínico, o grau de urgência do paciente para receber o medicamento prescrito, bem como que sejam explicitados os benefícios e riscos esperados, com base na melhor literatura científica disponível (6). As análises destas solicitações possibilitam intervenções que auxiliam na escolha terapêutica mais segura e eficaz, redução dos custos para a instituição, além de possibilitar a inclusão e exclusão de medicamentos quando necessário.

Sendo assim, o presente estudo pretende analisar as solicitações de medicamentos não padronizados enviados ao Setor de Farmácia para subsidiar a Comissão de Farmácia e Terapêutica na atualização da lista de medicamentos padronizados da instituição.

\section{Métodos}

Estudo descritivo, retrospectivo caracterizado como exploratório e realizado através da análise de 153 formulários de solicitações de medicamentos não padronizados enviados ao setor de farmácia do Hospital Universitário Dr. Miguel Riet Correa Jr no período de 01 de agosto de 2018 a 31 de dezembro de 2018. Esta pesquisa seguiu os preceitos trazidos pela resolução 466/12 do Conselho Nacional de Saúde e foi aprovada pelo Comitê de Ética em Pesquisa (CEP) da FURG em 29/01/2020 com o n ${ }^{\circ}$ CAAE 24643119.7.0000.5324.

Identificaram-se os seguintes desfechos a partir das solicitações: intervenções farmacêuticas (troca dos medicamentos não padronizados solicitados por medicamentos padronizados, que apresentam a mesma indicação/ação farmacológica e com indicação clínica baseada em evidências científicas para o caso analisado), altas dos pacientes antes da finalização do processo de compra, os medicamentos que foram aprovados e liberados para os pacientes internados. E os medicamentos que não foram comprados por falta no mercado e não aprovados pelo Hospital. 
Também se identificou os medicamentos não padronizados mais pedidos e as clínicas médicas que mais solicitaram (Clínica Médica (CM), Pediatria (PED), Unidade de Terapia Intensiva Geral (UTI GERAL), Serviço de Pronto Atendimento (SPA), Unidade de Terapia Intensiva Pediátrica (UTI PED), Clínica Cirúrgica (CC), Maternidade, Bloco Cirúrgico (BLOCO)).

Como critérios de inclusão foram consideradas as solicitações preenchidas por médicos e residentes, no período de 01 de agosto de 2018 a 31 de dezembro de 2018, com informações do leito, número do registro, quadro clínico com justificativa para aquisição do medicamento não padronizado, nome do medicamento, dose, posologia e tempo provável de uso. E como critério de exclusão, formulários encaminhados à farmácia fora do período de estudo, compreendido entre 01 de agosto de 2018 a 31 de dezembro de 2018 e preenchidos de modo incompleto.

Os dados clínicos, informações dos medicamentos não padronizados, nome da clínica solicitante e os desfechos das solicitações foram compilados em planilha eletrônica. Os dados coletados foram analisados no programa Microsoft Excel e expressos em gráficos e tabelas.

\section{Resultados}

Foram analisados 153 formulários de solicitações de medicamentos não padronizados, com uma média aproximada de 30 solicitações/mês e um total de 72 medicamentos diferentes. Dentre esses os mais requisitados foram: $11(7,19 \%)$ solicitação de alprazolam $1 \mathrm{mg}$ comprimido, $9(5,88 \%)$ de beclometasona $50 \mathrm{mcg}$ spray oral, $8(5,23 \%)$ de salbutamol 100 mcg spray oral e 7 (4,58\%) de vitamina do complexo B injetável. A tabela 1 demonstra os 31 medicamentos mais solicitados.

Tabela 1 - Frequência dos 31 medicamentos mais solicitados.

\begin{tabular}{|c|c|c|}
\hline Medicamentos & n (153) & $\%$ \\
\hline Alprazolam 1mg cp & 11 & 7.19 \\
\hline Beclometasona $50 \mathrm{mcg} / \mathrm{dose}$ spray oral & 9 & 5.88 \\
\hline Salbutamol 100 mcg spray oral & 8 & 5.23 \\
\hline Vitamina do Complexo B Injetável & 7 & 4.58 \\
\hline Omeprazol Magnésio 20mg cp rev & 6 & 3.92 \\
\hline Beclometasona 200mcg cáps oral & 4 & 2.61 \\
\hline Pregabalina 75mg cáps & 4 & 2.61 \\
\hline Hidralazina 25mg drágea & 4 & 2.61 \\
\hline Metadona 10mg cp & 4 & 2.61 \\
\hline Polimixina B 500.000UI suspensão & 4 & 2.61 \\
\hline Mupirocina 20mg/g & 3 & 1.96 \\
\hline Diosmin 450mg +Hesperidina 50mg cp rev & 3 & 1.96 \\
\hline Tansulosina $0,4 \mathrm{mg}$ cáps gelatinosa & 3 & 1.96 \\
\hline Dexpantenol 50mg/g gel oftalmico & 3 & 1.96 \\
\hline Ácido Ursodesoxicolico 50mg cp & 2 & 1.31 \\
\hline Albendazol $40 \mathrm{mg} / \mathrm{mL}$ suspensão & 2 & 1.31 \\
\hline Fenazopiridina 200mg drágea & 2 & 1.31 \\
\hline Fexofenadina 180mg cp & 2 & 1.31 \\
\hline Hidroxicloroquina 400mg cp rev & 2 & 1.31 \\
\hline Lorazepam $2 \mathrm{mg}$ cp & 2 & 1.31 \\
\hline Budesonida 32mcg spray nasal & 2 & 1.31 \\
\hline Lamotrigina 100mg cp & 2 & 1.31 \\
\hline Dimalato de Magnésio 150mg cáps & 2 & 1.31 \\
\hline Hialuronato de sódio $0,15 \%$ solução & 2 & 1.31 \\
\hline Brometo Ipratropio $20 \mathrm{mcg} / \mathrm{dose}$ spray & 2 & 1.31 \\
\hline Pamidronato 90mg & 2 & 1.31 \\
\hline Oxcarbazepina $6 \%$ suspensão oral & 2 & 1.31 \\
\hline Prednisolona 1\% colírio & 2 & 1.31 \\
\hline
\end{tabular}




\section{Quetiapina 25mg cp}

1.31

Vaselina Sólida Estéril 100\%

1.31

2

2

Legenda: cp: comprimido; cp rev: comprimido revestido, cáps: cápsula

continuação

Após a análise destas solicitações, pelos farmacêuticos clínicos, obtiveram-se os seguintes resultados: $110(71,90 \%)$ pedidos foram liberados, $16(10,46 \%)$ não foram aprovados para compra, $13(8,50 \%)$ sofreram intervenções farmacêuticas com substituição por medicamentos padronizados, $12(7,84 \%)$ não foram atendidos porque o paciente obteve alta antes da finalização da análise, $2(1,31 \%)$ pedidos não foram atendidos por descontinuidade do produto no mercado (Figura 1).

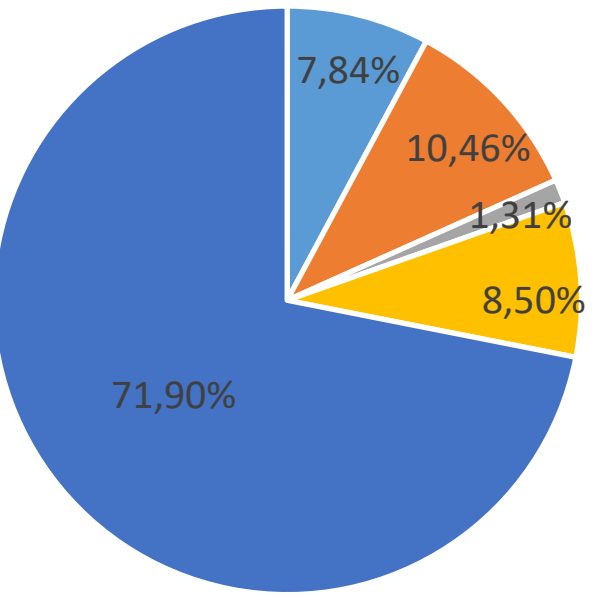

- Alta hospitalar antes da análise

- Não aprovados pelo Setor de Suprimentos

- Falta do medicamento no mercado

- Intervenções farmacêuticas

Figura 1 - Resultados das solicitações de medicamentos não padronizados.

No estudo também foram demonstrados quais as clínicas médicas da instituição que mais realizaram solicitações de medicamentos não padronizados (Figura 2).

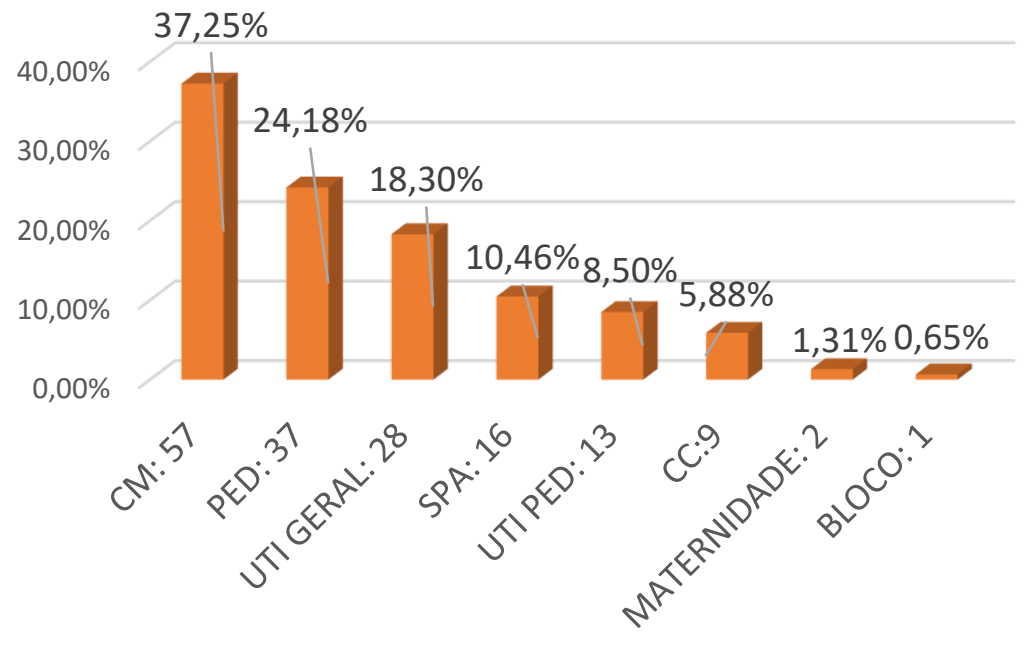

Figura 2 - Porcentagem de clínicas que encaminharam solicitações de medicamentos não padronizados. 


\section{Discussão}

Com a apresentação dos medicamentos mais solicitados é possível levá-los à Comissão de Farmácia e Terapêutica e discutir a possibilidade de inclusão destes medicamentos na lista de padronizados da instituição. No nosso estudo o medicamento alprazolam $1 \mathrm{mg}$ comprimido com $11(7,19 \%)$ solicitações é um candidato a inclusão nessa lista pois foi o mais solicitado. Assim como discutido por Santana et al. (2014), o impacto econômico dessas alterações deve ser levado em consideração, pois as decisões baseadas em evidências devem ser adaptadas aos recursos financeiros disponíveis, levando em conta as necessidades dos pacientes (7).

Os medicamentos alprazolam $1 \mathrm{mg}$ comprimido, beclometasona 50mcg spray oral, salbutamol $100 \mathrm{mcg}$ spray oral, foram os medicamentos não padronizados mais requeridos. Trabalhos análogos convergiram para resultados semelhantes ao encontrado neste estudo, considerando a finalidade de uso dos medicamentos não padronizados. Apesar dos diferentes contextos, os medicamentos do sistema nervoso central e do sistema respiratório foram os mais solicitados $(8,9)$.

Mesmo com a maioria dos pedidos atendidos, 110 (71,9\%), devemos considerar que os medicamentos prescritos fora da lista de padronização resultam na demora do início no tratamento ou uso do medicamento nem ser iniciado, devido a dificuldades de aquisição no serviço público (9).

Outras $13(8,50 \%)$ solicitações sofreram intervenções pelos farmacêuticos: substituição por medicamentos padronizados quando possível, o que demonstra que a prática farmacêutica no processo do uso racional do medicamento gera resultados positivos para o paciente e para os recursos investidos. Já que são necessárias estratégias de ação frente a prescrição de medicamentos que não estejam incluídos na padronização (10-12). A presença deste profissional nesta análise é de especial relevância, pois possui uma posição privilegiada para dimensionar o quantitativo de itens disponível no hospital e exerce atividades gerenciais e clínicas que são favorecidas pela racionalização da oferta (13).

Quando avaliamos as clínicas que mais solicitaram, observamos que a Clínica Médica que atende um público heterogêneo com patologias diversas foi a clínica que mais solicitou (37,25\%). Seguida pela Clínica Pediátrica (24,18\%) que apresenta um público suscetível a reações adversas e necessidade de adequações de formas farmacêuticas. Isto porque a clínica pode demandar mais ou melhores opções terapêuticas, tendo em vista a complexidade do cuidado oferecido e o perfil da população atendida. Desse modo ampliar o número de medicamentos oferecidos pode contar com justificativa não apenas quanto à adequação, mas quanto à necessidade (13).

\section{Limitações do estudo}

A falta da adesão da equipe médica para o envio das solicitações de medicamentos nãopadronizados para o setor de farmácia pode ter subestimados os dados apresentados. Este estudo descreveu apenas o perfil dos medicamentos não padronizados solicitados no hospital Dr. Miguel Riet Correa Jr, não é possível estender os resultados obtidos para todos os hospitais universitários.

\section{Conclusão}

O estudo demonstrou a importância da elaboração de uma lista de medicamentos não padronizados dentro de uma instituição, pois a partir dela é possível revisar a lista de padronizados e sugerir a inclusão de novos medicamentos a partir da identificação 
daqueles medicamentos não padronizados mais solicitados.

Também se concluiu que o farmacêutico possui ação relevante na análise destas solicitações porque garante o uso racional dos medicamentos e o gerenciamento de novas aquisições dentro do orçamento do hospital, gerando economia significativa nos gastos hospitalares e melhora no cuidado ao paciente.

\section{Referências}

1. Braga RJF. ABC da Farmácia Hospitalar. 1 ed. São Paulo: Editora Atheneu; 2014.

2. Ferracini FT, Filhos WMB. Prática Farmacêutica no Ambiente Hospitalar. 2 ed. São Paulo: Editora Atheneu; 2010.

3. Moura AA, Caparroti AB, dos Santos FN, Menezes JC, Luca R, Clemente SF et al. Gestão das solicitações de medicamentos não padronizados como ferramenta de qualidade da assistência ao paciente. Rev. Qualidade HC 2011; 2:79-82.

4. Ministério da Saúde (Brasil). Secretaria de Ciência,Tecnologia e Insumos Estratégicos. Departamento de Assistência Farmacêutica e Insumos Estratégicos. Formulário terapêutico nacional 2010: Rename 2010. 2 ed. Brasília: Ministério da Saúde; 2010.

5. Primo LP, Carvalho E, Lima AEU, Gandolfi CBT, Padula KM, Bózoli LFB, Abramovícius AC, Dallora MELV, Pazin-Filho A. Comissão de farmácia e terapêutica em um hospital de ensino. Medicina (Ribeirão Preto) 2015; 48(1):27-32.

6. Comissão Intergestores Tripartite (Brasil). Resolução no 29 de 26 de janeiro de 2017. Apresentação de justificativa para a prescrição de medicamento (s) não padronizado (s) no Sistema Único de Saúde (SUS) e centralização de dados. Diário Oficial [da] República Federativa do Brasil. 2017 nov. 01; Seção 1.p 56.

7. Santana RS, Jesus EMS, Santos DG, Lyra Júnior DP, Leite SN, Silva WB. Indicadores da seleção de medicamentos em sistemas de saúde: uma revisão integrativa. Rev Panam Salud Publica 2014; 35(3): 228-34.

8. Girotto E, Silva PV. A prescrição de medicamentos em um município do Norte do Paraná. Rev bras epidemiol 2006; 9(2): 226-234.

9. Araújo PTB, Uchôa SAC. Avaliação da qualidade da prescrição de medicamentos de um hospital de ensino. Ciênc Saúde Colet 2011;16(1): 1107-1114.

10. Bonal J, Castro I. Manual de formación para farmacéuticos clínicos. Madrid:Díaz de Santos; 1989.p. 48-54, p.149-56.

11. Puigventos F, Ventayol P, Cervera M, Ginés J, Delgado O. Evaluación de fármacos en el hospital: ¿Medicina basada en la evidencia o medicina basada en la eficiencia? Farm Hosp 2002; 131:14-21.

12. Conselho Nacional de Saúde (Brasil). Resolução no 338, de 6 de maio de 2004. Aprovar a Política Nacional de Assistência Farmacêutica. Diário Oficial da União. Brasília, 6 mai 2004; Seção1.

13. Magarinos-Torres R, Pagnoncelli D, Cruz-Filho AD, Osorio-de-Castro CGS. Vivenciando a seleção de medicamentos em hospital de ensino. Rev Bras Educ Med 2011; 35(1):77-85. 